\title{
Stereocontrolled Synthesis of (-)-Kainic Acid from trans-4-Hydroxy-L-Proline
}

\section{Jean-François Poisson,* Arturo G. Orellana and Andrew E. Greene}

This supporting information contains:

General experimental methods $\quad \mathrm{S} 1$

Experimental procedure for compound $\mathbf{7 b} \quad \mathrm{S} \quad \mathrm{S} 2$

Experimental procedure for compound $9 \quad \mathrm{~S} 2$

1H NMR spectrum for compound $7 \mathbf{b} \quad$ S4

13C NMR spectrum for compound 7b $\quad$ S5

Ortep diagram for compound $6 \quad$ S6

Reactions were generally carried out under argon in oven-dried glassware at $110^{\circ} \mathrm{C}$. Standard inert atmosphere techniques were used in handling all air and moisture sensitive reagents. Dry THF was obtained by filtration trough activated molecular sieves; dry $\mathrm{CH}_{2} \mathrm{Cl}_{2}$ by filtration through activated aluminum oxide. Hexamethylphosphoramide (HMPA) and methyl bromoacetate were distilled from calcium hydride. Thin-layer chromatography was performed on $(0.2 \mathrm{~mm})$ silica sheets, which were visualized under ultraviolet light and by heating the plate after treatment with either molybdophosphoric acid in ethanol, a $p$-anisaldehyde staining solution $(80 \mathrm{~mL}$ of $95 \%$ ethanol, $2.9 \mathrm{~mL}$ of sulfuric acid, $0.86 \mathrm{~mL}$ of acetic acid, $2.1 \mathrm{~mL}$ of $p$ -

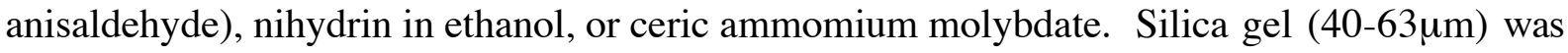
employed for flash column chromatography. A Fourier transform infrared spectrometer (FTIR) was used to record IR spectra (neat or as thin films of a dichloromethane or ether solution of the compound on sodium chloride discs). ${ }^{1} \mathrm{H}$ NMR and ${ }^{13} \mathrm{C}$ NMR spectra were recorded at 300 $\mathrm{MHz}$ and $75 \mathrm{MHz}$, respectively. All shifts for ${ }^{1} \mathrm{H}$ spectra were referenced to the residual solvent peak. Mass spectra were recorded using either DCI (ammonia/isobutane 63/37) or ESI techniques. Microanalyses were performed within our department. 


\section{(2S,3R,4R )-1-Benzyl 2-Methyl 3-(2-Acetoxyethyl)-4-hydroxypyrrolidine-1,2-}

dicarboxylate $\mathbf{( 7 b )}$. Acetyl chloride $(0.118 \mathrm{~mL}, 1.66 \mathrm{mmol})$ was added dropwise to a solution of the Cbz derivative 7a (448 mg, $1.39 \mathrm{mmol})$ and collidine $(0.366 \mathrm{~mL}, 2.75 \mathrm{mmol})$ in $10 \mathrm{~mL}$ of $\mathrm{CH}_{2} \mathrm{Cl}_{2}$ at $-78{ }^{\circ} \mathrm{C}$ under Argon. The reaction was stirred for $2.0 \mathrm{~h}$ at $-78{ }^{\circ} \mathrm{C}$, allowed to warm slowly to $0{ }^{\circ} \mathrm{C}$ and then quenched by the addition of water. The aqueous phase was extracted with EtOAc, which was washed with brine and dried over $\mathrm{MgSO}_{4}$. The volatiles were removed under reduced pressure. The crude product was purified by flash silica gel chromatography (eluent EtOAc/hexane, 1/1) to provide acetate $7 \mathbf{b}(415 \mathrm{mg}, 82 \%)$ as a colorless oil: IR 3444, 2952, 1730, $1698 \mathrm{~cm}^{-1} ;{ }^{1} \mathrm{H}$ NMR (300 MHz, $\mathrm{CDCl}_{3}$, mixture of rotamers) $\delta$ 1.79-1.94 (m, 1H), $2.00(\mathrm{~s}, 1.5 \mathrm{H}), 2.01(\mathrm{~s}, 1.5 \mathrm{H}), 2.03-2.10(\mathrm{~m}, 1 \mathrm{H}), 2.19-2.29(\mathrm{~m}, 1 \mathrm{H}), 3.27$ (br s, $1 \mathrm{H}), 3.49$ (s, $1.5 \mathrm{H}), 3.56-3.76(\mathrm{~m}, 2 \mathrm{H}), 3.73(\mathrm{~s}, 1.5 \mathrm{H}), 4.04-4.14(\mathrm{~m}, 3 \mathrm{H}), 4.27(\mathrm{~m}, 1 \mathrm{H}), 4.92(\mathrm{~A}$ of $\mathrm{AB} \mathrm{q}, J=$ $12.3 \mathrm{~Hz}, 0.5 \mathrm{H}$ ), 5.08 (br s, $1 \mathrm{H}), 5.15$ (B of AB q, $J=12.3 \mathrm{~Hz}, 0.5 \mathrm{H}), 7.24-7.30(\mathrm{~m}, 5 \mathrm{H}) ;{ }^{13} \mathrm{C}$ NMR (75 MHz, $\mathrm{CDCl}_{3}$, mixture of rotamers) $\delta$ 20.6, 20.7, 25.3, 45.9, 46.7, 51.9, 52.2, 54.9, $55.3,62.3,62.4,62.6,62.8,67.1,67.2,69.5,70.4,127.7,127.9,128.2,128.3,135.9,136.2$, 154.3, 154.7, 157.0, 170.9, 171.0, 172.7, 172.9; $\mathrm{MS}\left(\mathrm{ESI}^{+}\right) \mathrm{m} / z 366(\mathrm{M}+\mathrm{H})^{+}, 388(\mathrm{M}+\mathrm{Na})^{+}$, $404(\mathrm{M}+\mathrm{K})^{+} ;$HRMS $\left(\mathrm{ESI}^{+}\right)$calcd for $\mathrm{C}_{18} \mathrm{H}_{23} \mathrm{NO}_{7}+\mathrm{Na}^{+}: 388.1372$. Found : 388.1375 .

\section{(2S,3R,4R)-1-(Benzyloxycarbonyl)-3-(2-hydroxyethyl)-4-(tosyloxy)pyrrolidine-2-}

carboxylic Acid (9). Aqueous $\mathrm{NaOH}(3 \mathrm{~N}, 2.3 \mathrm{~mL}, 6.9 \mathrm{mmol}$ ) was added to a solution of tosylate $8(359 \mathrm{mg}, 0.69 \mathrm{mmol})$ in $3 \mathrm{~mL}$ of THF. After being stirred for $24 \mathrm{~h}$, the reaction mixture was diluted with water and extracted twice with ether. The aqueous phase was then acidified to $\mathrm{pH} 2$ with $1 \mathrm{~N} \mathrm{HCl}$. This aqueous phase was extracted with ethyl acetate, which was washed with brine and dried over $\mathrm{MgSO}_{4}$. The volatiles were removed under reduced pressure to give acid 9 (296 mg, 92\%) as a white solid: $\mathrm{mp} 116-117^{\circ} \mathrm{C}$ (racemic compound: lit. ${ }^{\text {5aa }} 116-118$ $\left.{ }^{\circ} \mathrm{C}\right) ;[\alpha]_{\mathrm{D}}^{20}+5.5\left(c\right.$ 1.0, $\left.\mathrm{CHCl}_{3}\right)$; IR 3441, 2932, $1713 \mathrm{~cm}^{-1} ;{ }^{1} \mathrm{H}$ NMR (300 $\mathrm{MHz}, \mathrm{CDCl}_{3}$, mixture of rotamers) $\delta$ 1.76-1.90 (m, 2H), $2.39(\mathrm{~s}, 2 \mathrm{H}), 2.42(\mathrm{~s}, 1 \mathrm{H}), 2.60-2.72(\mathrm{~m}, 1 \mathrm{H}), 3.45-$ $3.76(\mathrm{~m}, 4 \mathrm{H}), 4.00(\mathrm{~d}, J=9.9 \mathrm{~Hz}, 0.33 \mathrm{H}), 4.04(\mathrm{~d}, J=9.9 \mathrm{~Hz}, 0.67 \mathrm{H}), 4.99-5.13(\mathrm{~m}, 3 \mathrm{H}), 7.24-$ $7.36(\mathrm{~m}, 7 \mathrm{H}), 7.72(\mathrm{~d}, J=8.4 \mathrm{~Hz}, 1 \mathrm{H}), 7.75(\mathrm{~d}, J=8.4 \mathrm{~Hz}, 1 \mathrm{H}) ;{ }^{13} \mathrm{C} \mathrm{NMR}\left(75 \mathrm{MHz}, \mathrm{CDCl}_{3}\right.$, mixture of rotamers) $\delta 21.2,22.2,29.4,29.6,53.1,53.3,60.1,68.1,68.4,80.6,81.2,128.2$, $128.3,128.4,128.5,128.8,129.0,129.1,130.6,130.7,133.8,136.4,136.5,146.0,146.1,154.6$, 
155.8, 177.1; MS (ESI $\left.{ }^{+}\right) m / z 486(\mathrm{M}+\mathrm{Na})^{+}$; Anal. Calcd for $\mathrm{C}_{22} \mathrm{H}_{25} \mathrm{NO}_{8} \mathrm{~S} \cdot 0.5 \mathrm{H}_{2} \mathrm{O}: \quad \mathrm{C}, 55.92$; H, 5.55; N, 2.96. Found: C, 56.08, H, 5.55, N, 2.96.

\section{(2S,3S,4S)-1-(Benzyloxycarbonyl)-4-(prop-1-en-2-yl)pyrrolidine-2,3-dicarboxylic}

Acid. The Jones oxidation reaction was performed as previously described. ${ }^{\text {aa }} 87 \%$ yield. $[\alpha]_{D}^{20}-39.7\left(c 1.0, \mathrm{CHCl}_{3}\right)$. The spectroscopic data were in full agreement with those reported. ${ }^{5 a a}$ (The product was obtained as a white solid, $\mathrm{mp} 53-55^{\circ} \mathrm{C}$ ) 


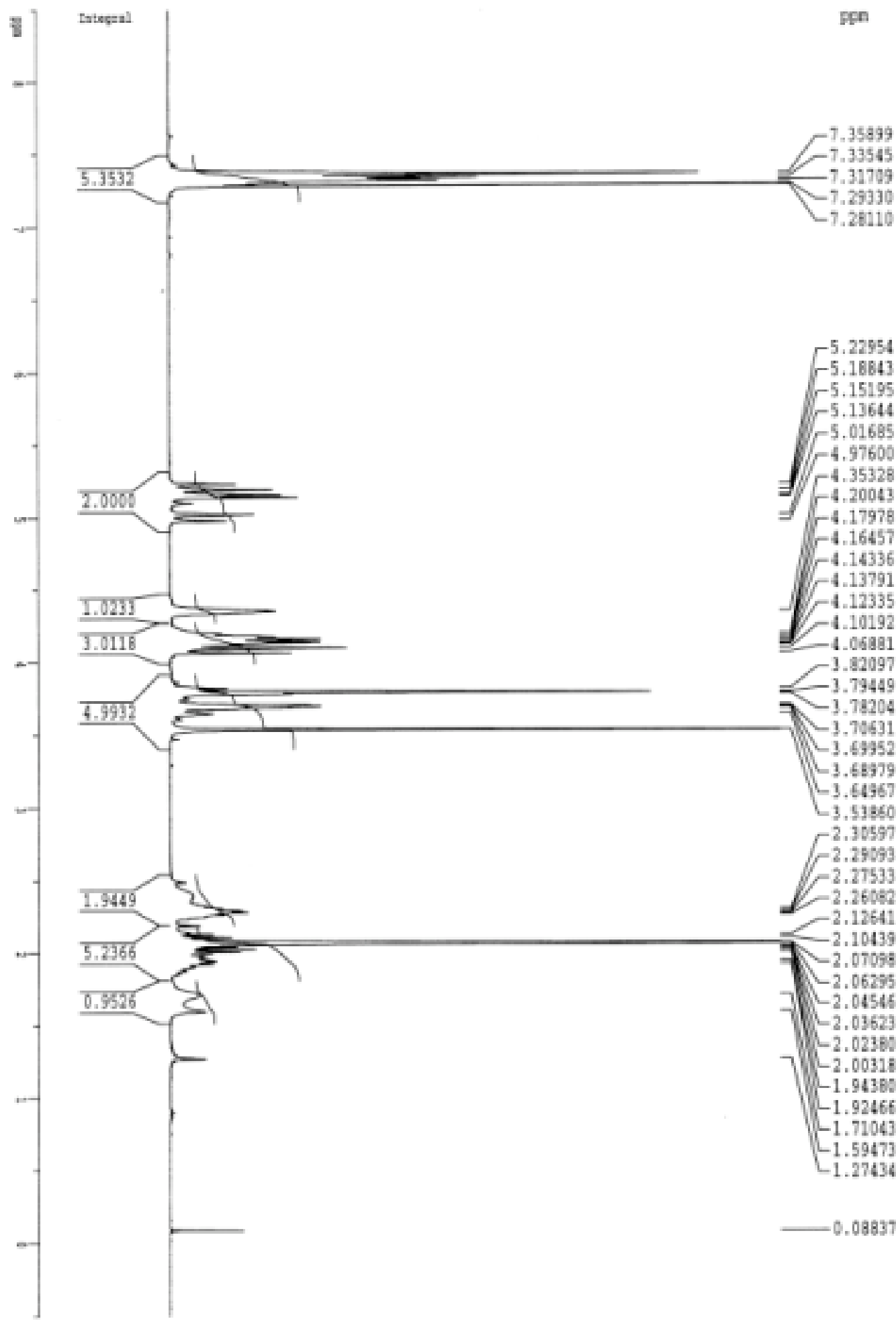




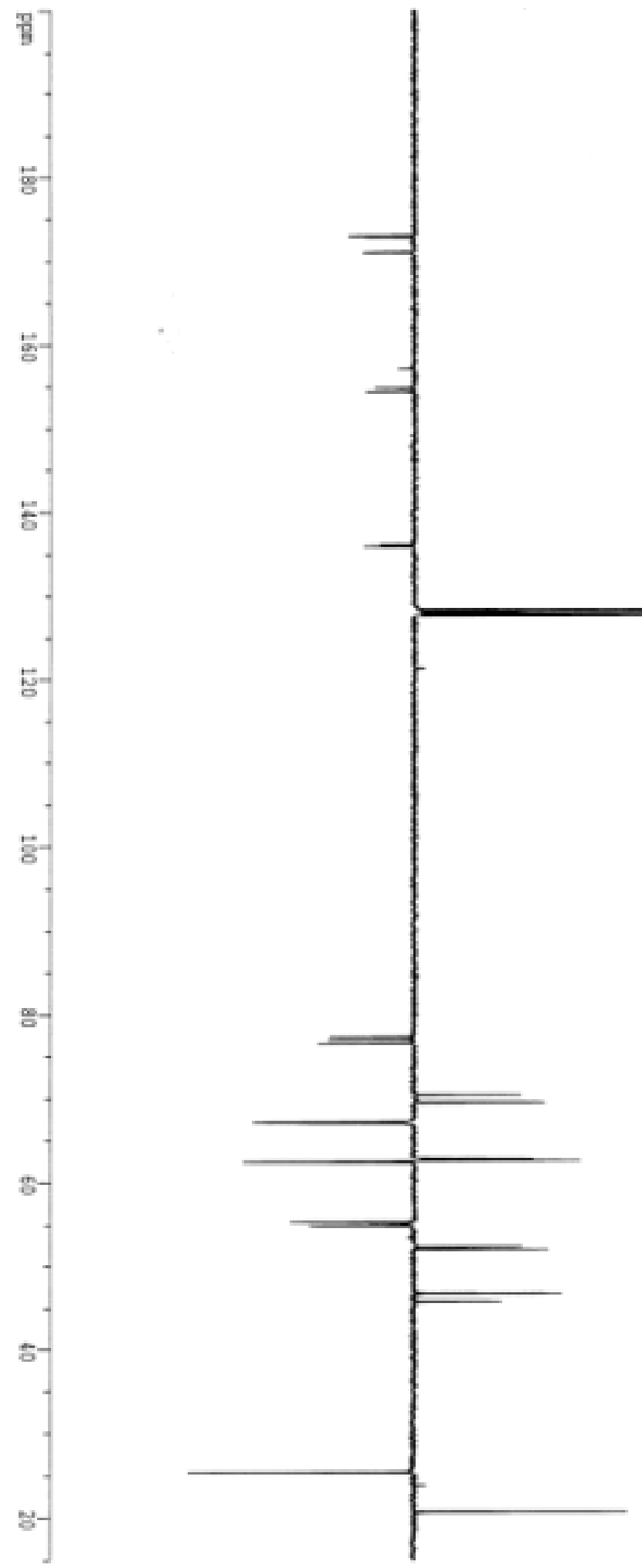

Ben

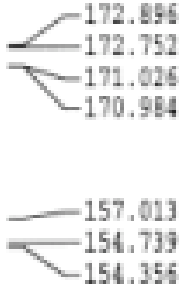

$-136.246$

135.949

128.311

128.262

127.859

$-127.692$

121.327

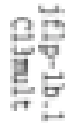

$\Gamma^{77.625}$

77.003

76.578

70.409

67.215

67.091

62.762

62.591

62.464

62.369

62.389
$-\quad 55.352$

$-54.915$

53.323
-52.265

53.3265
-51.969

51.964

-46.719
-65.860

45.860

25.322

23.817

20.755

- 20.625 
Ortep diagram of compound 6, (Probability set up at 25\%)

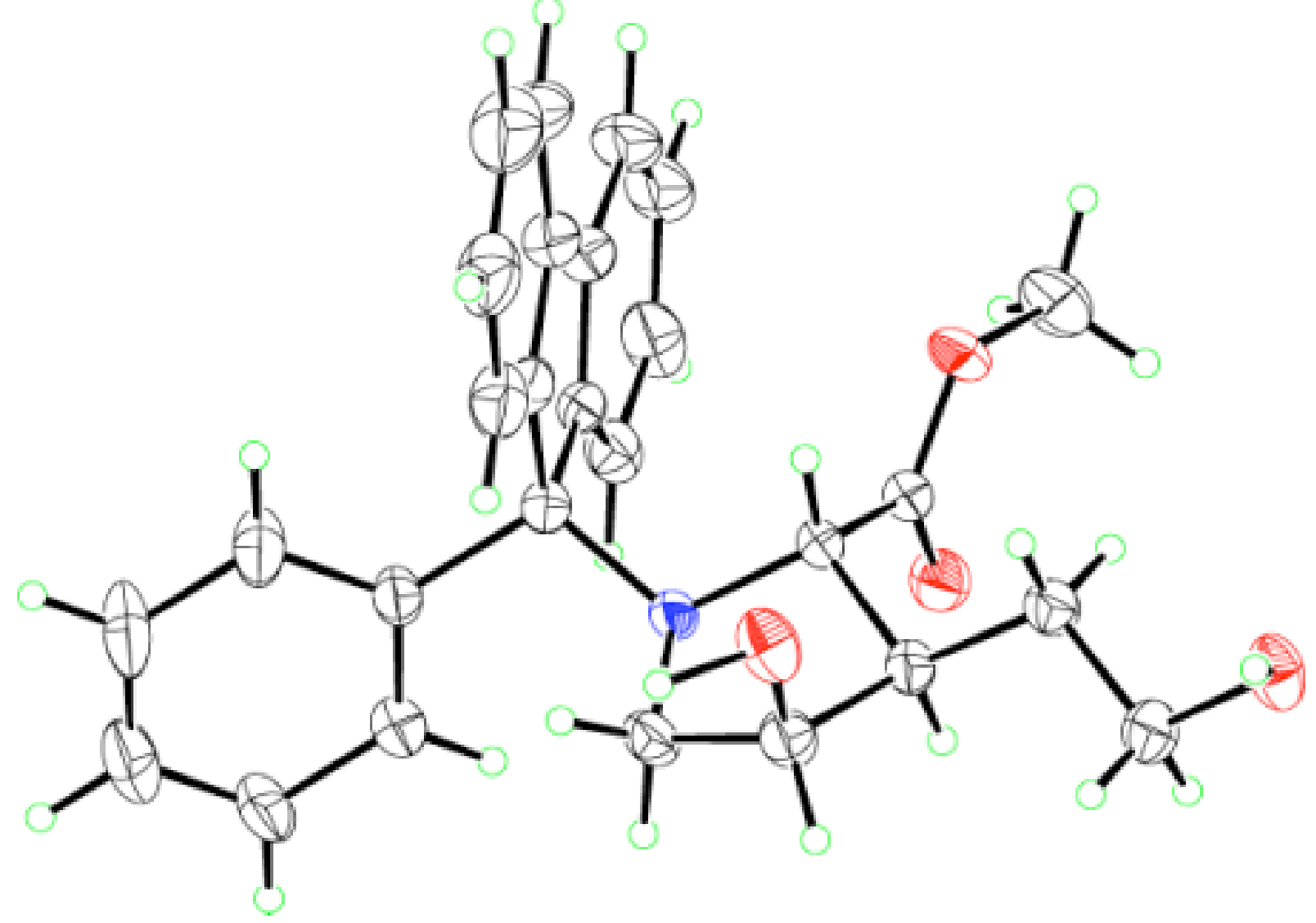

